\title{
Sobre as incertezas do tipo A e B e sua propagação sem derivadas: uma contribuição para a incorporação da metrologia contemporânea aos laboratórios de física básica superior \\ (On type $A$ and type $B$ uncertainties and its propagation without derivatives: a contribution to incorporate contemporary metrology to Physics' laboratories in higher education)
}

\author{
Paulo Lima Junior ${ }^{1}$ e Fernando Lang da Silveira
}

Instituto de Física, Universidade Federal do Rio Grande do Sul, Porto Alegre, RS, Brasil Recebido em 27/1/2011; Aceito em 23/2/2011; Publicado em 3/6/2011

\begin{abstract}
Após introduzir o conceito de incerteza, distinguindo procedimentos de avaliação da incerteza do tipo A e do tipo B, o presente artigo discute a lei de propagação da incerteza definida no Guia para Expressão da Incerteza da Medição, apresentando sua dedução e fundamentos. O objetivo deste trabalho é introduzir um procedimento alternativo que permite calcular a incerteza que se propaga das grandezas de entrada para a grandeza de saída sem referência explícita a derivadas parciais. Como o procedimento alternativo proposto é relativamente simples e compartilha o mesmo domínio de validade da própria lei de propagação da incerteza, é possível perceber seu valor para a introdução de conceitos e procedimentos metrológicos desde as primeiras atividades experimentais realizadas em laboratórios de física básica superior.
\end{abstract}

Palavras-chave: metrologia, incerteza, atividades experimentais, ensino superior.

After introducing the concept of uncertainty and procedures for evaluating type A and type B uncertainties, we discuss the law for propagation of uncertainty in the Guide to the Expression of Uncertainty in Measurement and its underlying assumptions. The main goal of this paper is to introduce an alternative procedure for estimating uncertainty that propagates from the input to output quantity without explicit reference to partial derivatives. As this alternative procedure is relatively simple and shares the same validity of the law for propagation itself, it is possible to perceive its value to the introduction of metrological concepts and procedures from the earliest experimental activities performed in higher education Physics' laboratories.

Keywords: metrology, uncertainty, laboratory activities, higher education.

\section{Introdução}

A metrologia é um campo de produção de conhecimento que diz respeito aos processos e procedimentos de medição em quaisquer atividades profissionais. Assim, ela aborda, por exemplo: o controle de qualidade dos produtos e insumos industriais, a condução de pesquisa básica, a produção de novas tecnologias, o desenvolvimento e a calibração de padrões de medida [1]. Por essa razão, trata-se de uma disciplina fundamental para a formação básica da maioria dos profissionais que cursam disciplinas de física experimental (ou outras disciplinas científicas que utilizem procedimentos de medida e análise quantitativa de resultados observacionais/experimentais), sobretudo os futuros bacharéis

\section{e licenciados em física.}

A confiabilidade dos resultados das medições é uma questão crítica no campo da metrologia. A esse respeito, há uma série de divergências históricas relacionadas às definições e aos procedimentos que permitem estimar erros e incertezas. Um passo fundamental em direção à superação dessas divergências foi dado na década de 1990 com a publicação de dois documentos: (1) Guia para Expressão da Incerteza da Medição [1]; e (2) Vocabulário Internacional de Metrologia [2]. Esses documentos são resultado do trabalho de especialistas indicados por sete organismos internacionais ${ }^{2}$ e estabelecem diretrizes amplamente aceitas para expressar e avaliar a confiabilidade dos resultados de uma medição.

\footnotetext{
${ }^{1}$ E-mail: paulolima@if.ufrgs.br.

${ }^{2}$ BIPM (Bureau International des Poids et Mesures), IEC (International Electrotechnical Commission), IFCC (International Federation of Clinical Chemistry), ISO (International Organization for Standardization), IUPAC (International Union of Pure and Applied Chemistry), IUPAP (International Union of Pure and Applied Physics) e OIML (International Organization of Legal Metrology). 
No campo da educação científica, alguns esforços vêm sendo realizados para esclarecer aspectos das novas diretrizes e do novo vocabulário da metrologia $[3,4]$ e incorporá-los ao ensino de física $[5,6]$. A saber, este artigo resulta da elaboração de um material didático diferenciado para a disciplina de mecânica experimental no Instituto de Física da Universidade Federal do Rio Grande do Sul (IF-UFRGS) em que se busca, entre outros propósitos, articular as atividades experimentais com contribuições do campo da metrologia.

Uma questão crítica, que surge da incorporação de metrologia às disciplinas introdutórias de física experimental, é a necessidade de se estabelecer estratégias que dispensem conhecimentos (matemáticos e físicos) e habilidades que os estudantes não adquiriram nos estágios iniciais do curso. Neste artigo, abordamos especificamente o problema clássico da propagação da incerteza - que diz respeito não somente às novas diretrizes da metrologia, mas às mais tradicionais teorias de erros e a necessidade de abordá-la sem referência explícita à derivação parcial.

Freqüentemente, em laboratórios didáticos e de pesquisa, usamos grandezas físicas conhecidas e medidas para inferir os valores de outra grandeza que não podemos - ou não desejamos - obter diretamente. Nesse tipo de situação em que a medição é indireta, a incerteza se propaga das grandezas conhecidas (grandezas de entrada) para a grandeza cujo valor se quer determinar (grandeza de saída). A incerteza da grandeza de saída $y$ obtida indiretamente a partir das grandezas de entrada $x_{1}, x_{2}, \ldots, x_{m}$, consideradas estatisticamente independentes, pelo modelo $y=f\left(x_{1}, x_{2}, \ldots, x_{m}\right)$, sob certas condições, pode ser obtida aproximadamente pela expressão

$$
\begin{aligned}
& u^{2}(y) \cong\left(\frac{\partial f}{\partial x_{1}}\right)^{2} u^{2}\left(x_{1}\right)+\left(\frac{\partial f}{\partial x_{2}}\right)^{2} u^{2}\left(x_{2}\right)+\ldots \\
& +\left(\frac{\partial f}{\partial x_{m}}\right)^{2} u^{2}\left(x_{m}\right)
\end{aligned}
$$

Nessa equação, $u\left(x_{j}\right)$ é a incerteza no valor da $j$ ésima grandeza de entrada e $u(y)$ é a incerteza no valor da grandeza de saída. Assim, a Eq. [1] traduz a chamada lei de propagação da incerteza [1]. Neste artigo, após introduzir o conceito de incerteza, deduzir essa lei e apontar os pressupostos que a precedem, é apresentado um método alternativo para obter a incerteza da medição da grandeza de saída sem referência explícita à derivação parcial.

\section{Introdução ao conceito de incerteza}

Quando se registra o resultado da medição de uma grandeza física, é obrigatório fazer alguma indicação quantitativa da qualidade desse resultado, de tal maneira que outras pessoas possam avaliá-lo e compará- lo com outros resultados [1]. O parâmetro quantitativo que expressa a confiabilidade do resultado de uma medição chama-se incerteza. Ela decorre da falta de precisão e exatidão no conhecimento que se tem a respeito do mensurando. Quanto maior for a incerteza de uma medida, menor será a confiabilidade que se deve atribuir a essa medida.

Toda grandeza estimada a partir de dados experimentais possui alguma incerteza na sua determinação. A esse respeito, existem fatores presentes em todos ou quase todos os processos de medição que implicam necessariamente a produção de incerteza. Por exemplo, todo processo de medição supõe a construção de um modelo em que o profissional lança mão de leis, idealizações e aproximações do seu campo de conhecimento para definir a grandeza física que está sendo medida e estabelecer um procedimento minimamente confiável que lhe permita estimar o valor dessa grandeza dentro de certos limites e para certos propósitos. Essa modelagem é realizada, ora de maneira intuitiva, ora de maneira sistemática, mas é sempre inerente ao processo de medição. Como todo processo de modelagem envolve idealizações e aproximações, mesmo se a medição puder ser realizada sob condições ideais, haverá alguma incerteza devido à própria definição da grandeza dentro do modelo. Esta quantidade é chamada incerteza definicional ou incerteza intrínseca ${ }^{[2]}$.

Por exemplo, quando decidimos medir o diâmetro de uma bolinha de metal, estamos considerando-a aproximadamente esférica (a rigor, ela pode ser oval, elipsoidal). Com efeito, trocar a medida do diâmetro da bolinha pela medida de semi-eixos equivale a trocar um modelo por outro. Assim, mesmo em situações experimentais extremamente elementares, para podermos responder à necessidade de definir o que estamos medindo, é indispensável lançar mão de um modelo. Estando todos os modelos sujeitos a idealizações e aproximações, a própria definição da grandeza medida é incerta.

Além do que foi dito até agora, há outros fatores que produzem incerteza no resultado de uma medição. Por exemplo: (1) variações entre observações repetidas sob condições aparentemente idênticas devidas à interferência de grandezas que não estão sendo controladas; (2) valores inexatos de constantes da natureza e outros parâmetros externos necessários para completar o processo de medição; (3) conhecimento imperfeito a respeito dos fenômenos envolvidos ou das condições de realização da medição; (4) viés subjetivo na leitura de escalas analógicas; (5) limites do instrumento com respeito à resolução ou ao seu limiar de discriminação. Todos esses fatores contribuem para que se tenha dúvida com respeito aos resultados de medição. 


\subsection{Incertezas padrão do tipo A e do tipo B}

A partir do GUM [1] é possível sustentar que toda incerteza é redutível a uma quantidade que pode ser expressa e interpretada como um desvio padrão independente da maneira como foi obtida. A incerteza que se expressa como desvio padrão é chamada incerteza padrão. ${ }^{3}$ O GUM reconhece dois tipos de incerteza (que equivalem a dois procedimentos de avaliação de incerteza). A incerteza que se obtém por análise estatística de uma série de observações chama-se incerteza do tipo $A$. A incerteza que se obtém por quaisquer outros métodos é chamada incerteza do tipo $B$. Tanto a incerteza do tipo A quanto a incerteza do tipo B podem ser interpretadas como desvios padrão.

São exemplos de avaliação da incerteza do tipo A o cálculo do desvio padrão da média de uma série de observações repetidas (por exemplo, do tempo de queda de um projétil) e o cálculo das incertezas nos parâmetros que se obtém do ajuste de curvas a dados experimentais [7]. Os procedimentos de avaliação da incerteza do tipo B são mais variados. De uma maneira geral, a avaliação da incerteza do tipo B deve levar em consideração todo o conhecimento disponível a respeito da propriedade ou do fenômeno em estudo [1]. Seja a incerteza obtida por um procedimento do tipo A ou do tipo B, ela sempre será interpretada como um desvio padrão.

\subsection{A avaliação da incerteza do tipo B}

Em contraste com a incerteza do tipo A, que envolve procedimentos já bastante conhecidos (tal como o cálculo do desvio padrão da média de uma série de observações feitas aproximadamente sob as mesmas condições), os procedimentos para estimar a incerteza do tipo B são um pouco mais elaborados e exigem maior atenção.

Conforme já foi dito, para estimar a incerteza do tipo B é necessário levar em consideração todas as informações disponíveis que estejam relacionadas à qualidade do resultado da medição. Uma maneira de se fazer isso é propor a priori uma distribuição de probabilidades (por exemplo, gaussiana, retangular, triangular, multinomial) que seja adequada para descrever a distribuição dos resultados de medição em torno do valor verdadeiro do mensurando ou do conjunto de valores verdadeiros que podem ser atribuídos a esse mensurando [1, 2]. Essa distribuição de probabilidades deve possuir pelo menos duas propriedades: (1) ela deve ser ajustável às informações prévias relevantes à determinação da qualidade do resultado da medição; (2) ela deve permitir a tradução dessas informações relevantes em uma quantidade que possa ser interpretada como desvio padrão.
Considere, por exemplo, a situação elementar em que desejamos determinar com uma régua o comprimento de um lápis. Apesar de ser muito simples, essa situação é importante porque o argumento desenvolvido para tratá-la pode ser facilmente estendido para qualquer medição que envolva escalas analógicas. Como, no exemplo do lápis e da régua, não há variabilidade perceptível dos resultados de medição, não será possível avaliar a incerteza por procedimentos do tipo A. Por outro lado, há pelo menos duas informações relevantes para avaliar a qualidade do resultado dessa medição: (1) o material com que foi feita a régua - geralmente réguas de plástico são fabricadas com calibração pior e com escalas menos bem definidas ou ilegíveis; e (2) a largura da menor divisão da escala - igual a $1 \mathrm{~mm}$.

Dado um valor verdadeiro $x_{v}$ qualquer para o comprimento do lápis, podemos considerar que a probabilidade de se obter cada resultado de medição $x$ satisfaça uma distribuição com densidade de probabilidade $f(x)$ uniforme e não-nula entre os valores $x_{v}-1 \mathrm{~mm}$ e $x_{v}+1 \mathrm{~mm}$ segundo a Fig 1a. Por outro lado, também poderíamos considerar, entre outras possibilidades, que a probabilidade de se obter cada resultado de medição $x$ satisfaz uma densidade de probabilidade triangular com os mesmos limites conforme a Fig. $1 \mathrm{~b}$.

(a) Distribuição uniforme

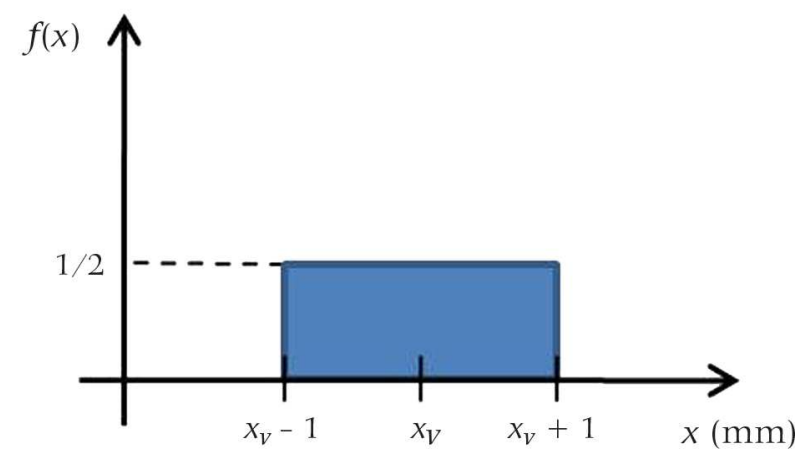

(b) Distribuição triangular

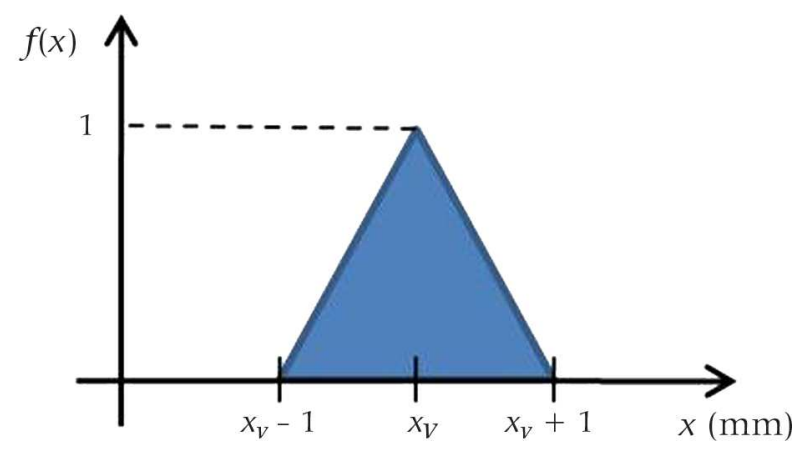

Figura 1 - Exemplos de funções densidade de probablidade na estimativa da incerteza do tipo B

\footnotetext{
${ }^{3} \mathrm{~A}$ rigor, nem toda a incerteza pode ser interpretada diretamente como desvio padrão. A incerteza que não se expressa como desvio padrão é chamada incerteza expandida. Entretanto, a incerteza expandida deve ser sempre igual à incerteza padrão multiplicada por um número real positivo, que é chamado fator de abrangência. Para o presente artigo, o conceito de incerteza expandida não é fundamental. Portanto, todas as ocorrências do conceito "incerteza" se referem exclusivamente à incerteza padrão.
} 
Sabendo que distribuições de probabilidade contínuas têm suas variâncias definidas por

$$
\sigma_{x}^{2}=\int_{-\infty}^{\infty}\left(x-x_{v}\right)^{2} f(x) d x
$$

em que $f(x)$ representa a função densidade de probabilidade, é possível calcular o desvio padrão de cada uma dessas duas distribuições. Para a distribuição uniforme, obtém-se $\sigma_{x, h}=1,0 \mathrm{~mm} / \sqrt{3} \cong 0,6 \mathrm{~mm}$. Para a distribuição triangular obtém-se $\sigma_{x, t}=1,0 \mathrm{~mm} / \sqrt{6} \cong$ 0,4 mm. Esses dois valores são exemplos de incertezas que resultam de uma avaliação do tipo B.

Como é possível perceber, esse processo de modelagem probabilística a priori que está implicado na avaliação da incerteza do tipo B é relativamente arbitrário (no sentido em que depende das escolhas feitas pelo sujeito responsável pelo processo de medição). Por isso, é fundamental que cada escolha na construção do modelo utilizado seja justificada com base em informações relevantes à determinação da qualidade dos resultados de medição. Por exemplo, levando em consideração que réguas de plástico são menos confiáveis que réguas de metal, poderíamos reservar a distribuição triangular para esta e a uniforme para aquela. Independente das escolhas feitas pelo sujeito responsável por avaliar a incerteza da medição, é fundamental destacar que o resultado de procedimentos semelhantes ao exemplificado nesta seção pode sempre ser interpretado como um desvio padrão.

\section{A propagação da incerteza}

Conforme foi antecipado, em laboratórios didáticos e de pesquisa estamos freqüentemente lidando com medições indiretas, ou seja, com processos de medição em que, a partir de um conjunto de grandezas cujos valores são conhecidos dentro dos limites de suas incertezas, desejase inferir o valor de outra grandeza (chamada grandeza de saída). Isso ocorre, por exemplo, quando determinamos a aceleração da gravidade a partir do período e comprimento de um pêndulo. Nesse tipo de situação em que a medição é indireta, a incerteza se propaga das grandezas de entrada para a grandeza de saída e a forma mais usual de estimar a incerteza no valor da grandeza de saída é substituir as melhores estimativas dos valores das grandezas de entrada e suas respectivas incertezas na lei de propagação da incerteza [1].

\subsection{Dedução da lei de propagação da incerteza}

Considere que $x_{i j}$ representa a $i$-ésima observação da $j$-ésima variável e que foram feitas $n$ observações para cada uma das $m$ variáveis de entrada. Assim, a cada conjunto $\left\{x_{i 1}, x_{i 2}, \ldots, x_{i m}\right\}$ de valores observados corresponde uma quantidade $y_{i}$ tal que $y_{i}=$ $\left(x_{i 1}, x_{i 2}, \ldots, x_{i m}\right)$. Considere também que $\bar{y}$ representa a média dessas quantidades e, ao mesmo tempo, a melhor estimativa do valor da grandeza $y$. Nesse caso, dado que a incerteza padrão $u(y)$ pode ser calculada a partir do desvio padrão da média de $y$, podemos escrever

$$
u^{2}(y)=\frac{1}{n(n-1)} \sum_{i=1}^{n}\left(y_{i}-\bar{y}\right)^{2}
$$

Sejam $\Delta x_{i j}=x_{i j}-\bar{x}_{j}$ e $\Delta y_{i}=y_{i}-\bar{y}$. Com efeito, na medida em que as variações $\Delta x_{i j}$ forem pequenas ao ponto do modelo matemático $y=f\left(x_{1}, x_{2}, \ldots, x_{m}\right)$ poder ser aproximado por um hiperplano em uma região que contenha essas variações, podemos substituir $y_{i}-\bar{y}$ na expressão anterior pelo termo de primeira ordem da série de Taylor

$$
\Delta y_{i}=\sum_{j=1}^{m} \frac{\partial f}{\partial x_{j}} \Delta x_{i j}+O\left(\Delta x^{2}\right) \cong \sum_{j=1}^{m} \frac{\partial f}{\partial x_{j}} \Delta x_{i j}
$$

Observe que todas as derivadas na expressão anterior e seguintes, pela definição das séries de Taylor, são avaliadas no ponto em que $x_{j}=\bar{x}_{j}, \forall j$. Substituindo a expansão [4] na expressão [3], obtemos

$$
\begin{array}{r}
u^{2}(y) \cong \sum_{j=1}^{m}\left(\frac{\partial f}{\partial x_{j}}\right)^{2} u^{2}\left(x_{j}\right)+ \\
2 \sum_{k>j}^{m} \sum_{j=1}^{m}\left(\frac{\partial f}{\partial x_{j}}\right)\left(\frac{\partial f}{\partial x_{k}}\right) \operatorname{cov}\left(x_{j}, x_{k}\right)
\end{array}
$$

Nessa expressão, $\operatorname{cov}\left(x_{j}, x_{k}\right)$ representa a covariância das médias das grandezas de entrada $x_{j}$ e $x_{k}$. Se as grandezas de entrada forem estatisticamente independentes e, portanto, suas covariâncias forem nulas, obtemos a lei de propagação da incerteza

$$
u^{2}(y) \cong \sum_{j=1}^{m}\left(\frac{\partial f}{\partial x_{j}}\right)^{2} u^{2}\left(x_{j}\right)
$$

Como é possível perceber, a forma mais simples da lei de propagação da incerteza carrega fundamentalmente os seguintes pressupostos: (1) a independência estatística das grandezas de entrada; (2) o modelo matemático $y=f\left(x_{1}, x_{2}, \ldots, x_{m}\right)$ pode ser aproximado por um hiperplano na região que contém as variações $\Delta x_{i j}$. Quando essas duas condições não forem completamente satisfeitas, é possível retomar a dedução da lei de propagação da incerteza introduzindo termos de ordem superior e termos com as devidas covariâncias entre as variáveis de entrada. Contudo, na maioria das situações usuais em laboratórios didáticos, a Eq. [6] resulta em excelente aproximação.

\subsection{Método alternativo para propagação da incerteza}

Os pressupostos que fundamentam a lei de propagação da incerteza implicam que exista uma função $g$ linear nas grandezas de entrada tal que $y \cong g\left(x_{1}, x_{2}, \ldots, x_{m}\right)$ 
em uma região que contenha as variações $\Delta x_{i j}$. Com isso, $g\left(x_{1}, x_{2}, \ldots, x_{m}\right)=a_{0}+\sum_{j=1}^{m} a_{j} x_{j}$. Acrescentando a hipótese de independência estatística e, portanto, de covariâncias nulas entre as grandezas de entrada, obtemos

$$
u^{2}(y) \cong \sum_{j=1}^{m} a_{j}^{2} u^{2}\left(x_{j}\right)
$$

Como a função $g\left(x_{1}, x_{2}, \ldots, x_{m}\right)$ é linear em cada uma das suas variáveis, os coeficientes $a_{j}$ podem ser calculados exatamente pela razão entre $\Delta g$ e $\Delta x_{j}$, mantido todo o resto constante

$$
a_{j}=\left(\frac{\Delta g}{\Delta x_{j}}\right)_{x_{k}=\bar{x}_{k}, \forall k \neq j}
$$

Como a aproximação linear é considerada satisfatória em qualquer região que contenha as variações $\Delta x_{i j}$, podemos fazer $\Delta x_{j}=u\left(x_{j}\right)$ e $g \cong y$. Com isso, a incerteza da grandeza de saída $u(y)$ pode ser estimada por

$$
u^{2}(y) \cong \sum_{j=1}^{m}\left(\Delta y_{j}\right)^{2}
$$

Nesta expressão, $\Delta y_{j}$ representa a variação que o modelo matemático $y=f\left(x_{1}, x_{2}, \ldots, x_{m}\right)$ experimenta quando é acrescentada uma quantidade $u\left(x_{j}\right)$ à $j$ ésima grandeza de entrada, mantendo-se todas as outras grandezas fixas em suas melhores estimativas $\bar{x}_{k}$.

\subsection{Exemplo de aplicação do método alterna- tivo}

O argumento apresentado nesta seção permitiu desenvolver um procedimento simples, relativamente geral (pois compartilha o mesmo domínio de validade da própria lei de propagação da incerteza) e, do ponto de vista educacional, potencialmente significativo (porque sua discussão supõe somente que os alunos possuam alguns conhecimentos rudimentares sobre representação gráfica de funções) que pode ser apresentado e discutido na sua quase totalidade desde a primeira atividade experimental do curso de graduação [8].

Para ver o procedimento em operação, considere, como exemplo, que desejamos determinar a aceleração da gravidade com um pêndulo de Kater [9] ao qual corresponde um pêndulo simples de comprimento $l$ dado por $(48,381 \pm 0,003) \mathrm{cm}$ e período $T$ dado por $(1,3964$ $\pm 0,0002)$ s. Nesse caso, considerando que seja possível determinar a aceleração da gravidade pela expressão $g=4 \pi^{2} l / T^{2}$, a incerteza $u(g)$ pode ser obtida da seguinte maneira:

1. Calcula-se a melhor estimativa do valor da grandeza de saída a partir das melhores estimativas dos valores das grandezas de entrada pelo modelo matemático $y=f\left(x_{1}, x_{2}, \ldots, x_{m}\right)$. Neste caso, $\bar{g} \cong 4 \pi^{2} \bar{l} / \bar{T}^{2} \cong 979,524 \mathrm{~cm} / \mathrm{s}^{2}$ (resultado expresso com um grande número de algarismos).

2. Em seguida, acrescenta-se a incerteza padrão ao valor de cada uma das grandezas de entrada. Os valores obtidos são inseridos novamente no modelo matemático para obter $\bar{y}+\Delta y_{j}$ em que $\Delta y_{j}$ é o incremento na grandeza de saída devido exclusivamente ao acréscimo de $u\left(x_{j}\right)$ ao valor da $j$-ésima grandeza de entrada.

$$
\begin{aligned}
\bar{g}+\Delta g_{l} \cong 4 \pi^{2}[\bar{l}+u(l)] / \bar{T}^{2} \cong 979,585 \mathrm{~cm} / \mathrm{s}^{2} . \\
\bar{g}+\Delta g_{T} \cong 4 \pi^{2} \bar{l} /[\bar{T}+u(T)]^{2} \cong 979,804 \mathrm{~cm} / \mathrm{s}^{2} .
\end{aligned}
$$

3. Enfim, as diferenças entre os valores obtidos no passo (2) e o valor obtido no passo (1) são elevadas ao quadrado e somadas para produzir o quadrado da incerteza na medição da grandeza de saída $u(y)$. No exemplo em questão, $u(g) \cong$ $\sqrt{\Delta g_{l}^{2}+\Delta g_{T}^{2}}=\sqrt{0,06^{2}+0,28^{2}} \cong 0,3 \mathrm{~cm} / \mathrm{s}^{2}$.

Assim, a melhor estimativa da aceleração da gravidade a partir desses dados é dada por $\bar{g}=979,5 \mathrm{~cm} / \mathrm{s}^{2}$ com incerteza $u(g)=0,3 \mathrm{~cm} / \mathrm{s}^{2}$.

\section{Conclusão}

Como é possível perceber, o método expresso na Eq. [9] compartilha o mesmo domínio de validade da lei de propagação da incerteza na Eq. [5] com a vantagem de não fazer referência explícita à derivação parcial, podendo, por isso, ser apresentado e discutido com os estudantes de graduação desde sua primeira atividade experimental.

Enfim, o método alternativo proposto neste artigo é uma ferramenta consistente com os pressupostos da lei de propagação da incerteza, podendo ser apresentado e discutido desde as primeiras atividades da física experimental superior.

\section{Agradecimento}

Agradecemos à Prof ${ }^{a}$ Maria Cristina Varriale do IMUFRGS pela leitura atenta e pelas sugestões apresentadas.

\section{Referências}

[1] Joint Commitee for Guides in Metrology (JCGM), Evaluation of Measurement Data: Guide to the Expression of Uncertainty in Measurement (GUM) (BIPM, Sèvres, 2008), $1^{\mathrm{a}}$ ed, 120 p. Disponível em www.bipm. org/en/publications/guides, acesso em 17/9/2010. 
[2] Joint Commitee for Guides in Metrology (JCGM). International Vocabulary in Metrology: Basic and General Concepts and Associated Terms (VIM). (BIPM, Sèvres, 2008), $3^{\text {a }}$ ed, 90 p. Disponível em Www.bipm. org/en/publications/guides, acesso em 17/9/2010.

[3] A. Cruz, E. Filipe, G. Almeida, J. Valadares e O. Pellegrino, Caderno Brasileiro de Ensino de Física 26, 125 (2009).

[4] J.H. Vuolo, Revista Brasileira de Ensino de Física 21, 350 (1999).

[5] A. Buffler, S. Alie and F. Lubben, The Physics Teacher 46, 539 (2008).
[6] P. Siegel, The Physics Teacher 45, 232 (2007).

[7] W.P. Silva, C.M.D.P.S. Silva, C.D.P.S. Silva e H.J.G. Silva, Revista Brasileira de Ensino de Física 21, 341 (1999).

[8] P. Lima Junior, F.L. Silveira e M.T.X. Silva, Medições Indiretas e Propagação da Incerteza (IF-UFRGS, Porto Alegre, 2011). Disponível em www.if .ufrgs.br/ $\sim$ lang/semderivadas.pdf, acesso em 28/2/2011.

[9] F.L. Silveira, Revista de Enseñanza de la Física 10, 29 (1995). 